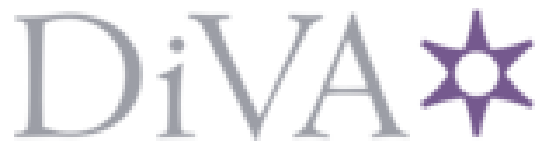

http://www.diva-portal.org

This is the published version of a paper presented at IEEE 11th International Conference on Communications (COMM), JUN o9-11, 2016, Bucharest, ROMANIA.

Citation for the original published paper:

Guo, Y., Yao, Y., Bai, G. (2016)

On Enhancement of Inter-Activity for Knowledge Sharing in eHealth.

In: 2016 INTERNATIONAL CONFERENCE ON COMMUNICATIONS (COMM 2016)

(pp. 247-250). IEEE

International Conference on Communications (ICC)

N.B. When citing this work, cite the original published paper.

Permanent link to this version:

http://urn.kb.se/resolve?urn=urn:nbn:se:bth-13302 


\title{
On Enhancement of Inter-Activity for Knowledge Sharing in eHealth
}

\author{
Yang Guo, Yong Yao and Guohua Bai \\ Blekinge Institute of Technology, 37179 Karlskrona, Sweden \\ Email: \{yang.guo, yong.yao, guohua.bai\}@bth.se
}

\begin{abstract}
Today, knowledge sharing raises as an important issue that challenges for the eHealth management system. It becomes one of the most demanding applications with references to the dynamic inter-activities among different health actors and the complex data structures involved in this application. In this paper, we suggest an activity theory based ontology model to scientifically represent various health actors in the eHealth system. The goal of the suggest model is to enhance the interactivities among these health actors for the efficient knowledge sharing purposes. We also develop a prototype software system based on the suggested ontology model. The survey results collected from the system users show the feasibility of the developed software system.
\end{abstract}

Index Terms-eHealth, knowledge sharing, activity theory.

\section{INTRODUCTION}

The proportion of elderly people (aged 65 or over) in the european countries is predicted to rise from $16.4 \%$ in 2004 to $29.9 \%$ in 2050 [1]. This prediction shows a critical problem that more elderly people in the future may suffer from chronic diseases such as diabetes. To alleviate this problem, a promising solution is to strain the personal healthcare services to assist in self-care using the eHealth system. This can be done by two main service-oriented operations, i.e., data collection and data processing. The data collection is usually accompanied through advancing novel technologies for patients (e.g., elderly people), for instance, the wearable and mobile systems [2]. However, the data processing is a very challenging issue in the form of how to coordinate the involved health actors for the self-care assistant purpose. Therefore, one needs to design and develop an efficient management system to exchange and update the information acquired at the individual health actor side. The corresponding process conducted in eHealth is known as knowledge sharing.

So far, there are a large amount of studies done on knowledge sharing in the eHealth area. For instance, the authors of [3] report a multi-viewpoint based model to represent the heterogeneous health actors by using ontology. In [4], the authors report on the problem of distributed knowledge sharing among patients and physicians. They also suggest a multiagent based method to overcome this problem. Clearly, the focus of these studies is laid on the representation of different health actors. The corresponding solution approach for knowledge sharing is mainly operated on two traditional heath actors. They are the care provides (e.g., healthcare center, doctors, nurses) and the care receivers (e.g., elderly people). Given the

978-1-4673-8197-0/16/\$31.00 (C) 2016 IEEE increasing demand for self-care on chronic health problems, the care receivers may require the external assistant from other health actors like, e.g., family members, neighbours, social care agencies, third party organizations. This situation gives rise to the need of the long term financial sustainability of the personal healthcare services. Subsequently, the ultimate motivation comes down to the development of solution approach to enhance the inter-activities among different health actors. The goal is to support more general knowledge sharing among the aforementioned various health actors, and thus improving the quality and efficiency of the assistant in self-care. To the best of our knowledge, there are few studies done so far along with this research line.

In this paper, we study the inter-activities among different health actors in a particular eHealth system. We suggest a new model to deal with these inter-activities for the efficient knowledge sharing purposes. This model is based on the activity theory and an ontological model. Based on this model, we also develop a prototype software system called Activity Information System. The rest of paper is as follows. Section II describes the main health actors existing in eHealth. Section III represents the activity theory ontology and its advantage for representing the inter-activities among various health actors. Section IV describes our developed prototype software system, and reports the survey results on the deployment of this system. Finally, the paper is concluded in Section V.

\section{System Model And Inter-activities}

We consider a particular eHealth system, which is advanced to provide the personal healthcare services to elderly people. In this system, six different health actors are taken into consideration. They are described below:

- Database for care receivers: it stores the personal information about the care receivers for the self-care assistant purposes. Examples of such information are the age, the suffered chronic diseases, and the relatives (i.e., family members). Further, the information about the associated care providers is also required to be recorded in this database.

- Database for care providers: similarly, this database needs to record the personal information about the care providers. Examples of such information are the time scheduling of care providers, the competence of care providers, the information about the assigned care receivers. 
- Instruments: they are referred to as the items used for personal healthcare services, for instance, a laboratory, the electronic health record, the X-rays and the relevant diagnostic knowledge. These items need to be integrated into a single base, which provides the uniform Application Programming Interfaces (APIs) to different categories of users such as healthcare providers and receivers.

- Community network: it is connected to different social elements like, e.g., healthcare providers, healthcare receivers, family relatives, healthcare centers, shopping centres, banks, TAXI, family members.

- Laws/rules: they are used by different people to know the relevant information about the particular social laws, contracts, and decisions.

- Labour division: it provides the prescribed social contact information for dealing with personal healthcare, together with the suitable methods to solve the particular conflict problem among different people.

In the following, an activity theory based ontology model is suggested to represent these health actors, together with the descriptions of inter-activities among them.

\section{INTER-ACTIVITIES IN EHEALTH}

In the previous work [5], we report on the feasibility of using the activity theory to represent the health actors in the eHealth system. In this section, we extend our studies done in [5]. Specifically, we adopt the activity theory to further investigate the inter-activities among various health actors existent in the above considered eHealth system.

\section{A. Activity theory ontology for knowledge sharing in eHealth}

The activity theory is a conceptual framework with the responsibility of representing the abstract knowledge existing in mind. By activity, it means the basic unit that preserves the essential quality behind any human practice. The key idea of this theory is to explain the inseparability of subjective and the objective from the human practice point of view [6]. The activity theory is initially coined by the authors of [7] and [8]. In [9], the authors further develop the activity theory by suggesting a triangle model. This model is used to recapitulate and visualize the components and relationships among them.

In accordance with the triangle model advanced in [9], we suggest an activity theory based ontology model to represent the health actors described in Section II, as shown in Fig. 1. The main advantage of this model is referred to as the flexibility and extendibility of representing various health actors for conducting knowledge sharing in the eHealth system. The detailed description about the suggest model is as follows.

In Fig. 1, there are three key activity units, i.e., care provider, care receiver and tool box. First, the care provider and the care receiver are defined to be the activity subject and activity object, respectively. The work activity of the care provider is then referred to as the assistant in the selfcare requested by a particular care receiver. The outcome of this work activity is connected to the process of dealing with the particular health problem suffered by the particular

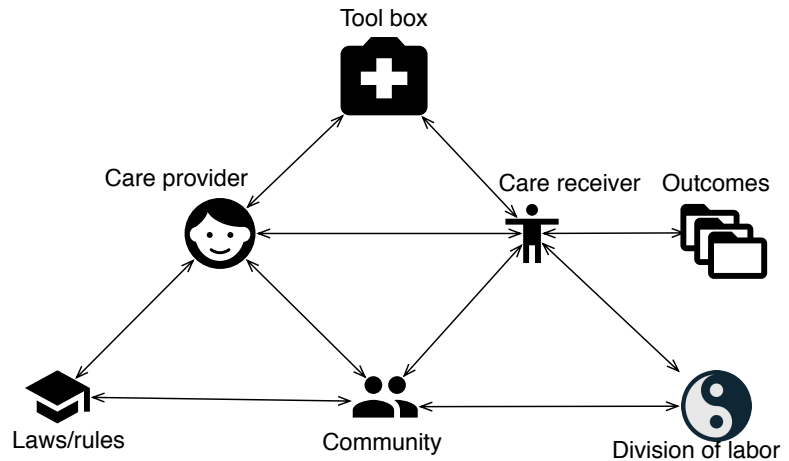

Fig. 1. Activity theory ontology model for knowledge sharing in eHealth

care receiver. To accomplish such process, one needs the particular instruments such as laboratory, medical devices (e.g., electronic health record, X-rays), relevant diagnostic knowledge, and the suitable psychological treatment methods. Further, all these instruments are provided by the tool box.

The functions of the above-described three key activity units are further extended by connecting them to another three activity units. The first unit is related to the community network, which is connected to both the care providers and receivers. This is because that different social contacts belonging to this community may need collaborate with care providers to give more efficient healthcare service to care receivers. The second unit is the division of labor, which is responsible for doing decision-making on the particular tasks in the eHealth system. Examples of tasks are assigning the competent physician for the patient, allocating the nurse to the patient, and arranging meetings between care providers and care receivers. Due to the limited resource on the care provider side, the division of labor is designed to connect to the community unit. The third unit is the associated with the prescribed rules, which give the particular constraints to different subjectives like, e.g., the legislate actions taken by care providers, the outcome evaluations, the reward criteria for physicians.

\section{B. Inter-activities among different health actors}

By using the suggested activity theory based ontology model, the eHealth system is capable to efficiently deal with various healthcare services. Accordingly, three typical cases are shown in Fig. 2. The detailed descriptions are as follows.

1) Case 1: a patient communicates with a nurse:

- (1): a patient stays at home and feels bad, and thus reporting the health status to a nurse, who is responsible for assisting this patient.

- (2): the nurse checks the health status reported by the patient. Depending on the particular condition of the health status, the nurse may give different feedbacks to the patient.

- (3): if the situation is not serious, the nurse may directly give the corresponding suggestion to the patient. If the situation is serious, the nurse immediately reports the 


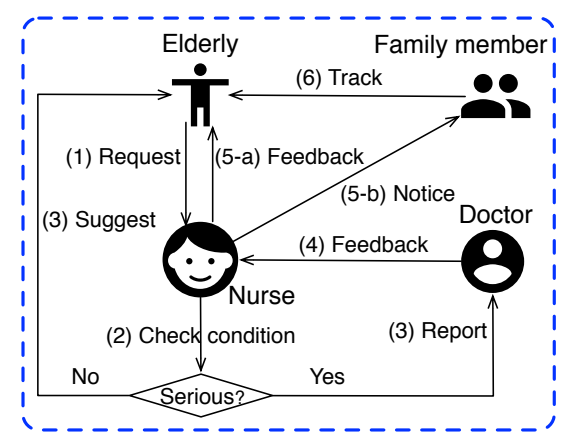

Case 1

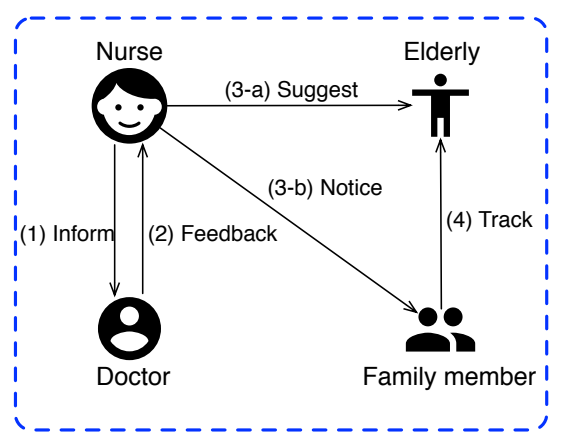

Case 2

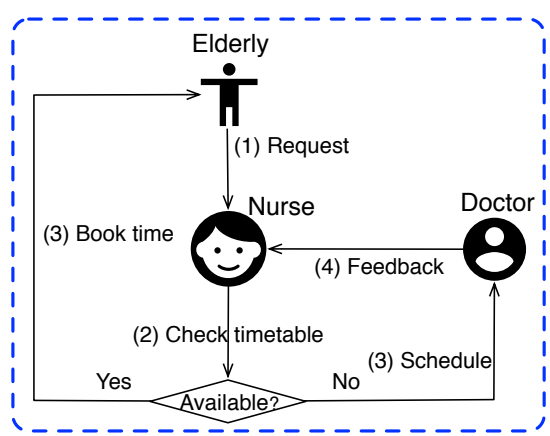

Case 3

Fig. 2. Three cases regarding personal healthcare using the activity theory based ontology model.

patient's health status to a doctor who is responsible for taking care of the patient.

- (4): the doctor makes a further investigation on the reported health status. The doctor also makes a prescription for the patient, and sends the prescription to the nurse.

- (5): the nurse provides the received prescription to the patient. Meanwhile, the nurse also sends the notice to the patient's family member.

- (6): the patient's family member could help the nurse to keep tracking the health status of the patient.

2) Case 2: a family member takes care of a patient:

- (1): a nurse checks the health status of a patient, which is reported by a patient's family member. The nurse observes that the patient's health status is not good, and thus informing a doctor who is responsible for taking care of the patient.

- $(2,3,4)$ : they are same to the steps $(4,5,6)$ described in case 1 .

3) Case 3: a patient makes an appointment to meet a doctor:

- (1): the patient sends an appointment request to a particular nurse who is responsible for assisting this patient.

- (2) the nurse checks the time availabilties of the corresponding doctor, and sends the suggested time slots to the doctor.

- (3): based on the received information about the suggested time slots and the appointment request, the doctor arranges a particular time slot to meet the patient. This particular time slot is then sent to the nurse.

- (4): once the nurse receives the arranged time slot for meeting from the doctor, the nurse informs this time slot to the patient and prepares for the meeting between the doctor and the patient.

\section{A Prototype Software System For Knowledge SHARING IN E-HEALTH}

Based on the suggested activity theory ontology, a prototype software system is developed to deal with the inter-activities for knowledge sharing in eHealth. This software system is called Activity Information System (AIS).

\section{A. System Design and Software Development}

Differing from the most existent healthcare systems, the AIS is designed to support different categories of healthcare activities (e.g., primary care, intensive care, home care, elderly care, disabled care, etc.). It allows different users to record, to track, and to share various information in eHealth like, e.g., the competence of a doctor, the time scheduling of a nurse, the chronic diseases of a patient.

An important goal of using the AIS is that the assistant in self-care services can be provided by either public/private healthcare organization or individual person (e.g., a doctor, a nurse, the patients' relatives). To achieve this goal, one demands for an uniform platform to share different information about the individual care actors. With regarding to the diversity of care actors, this platform needs to be simple for use, and to be independent from devices and operating system. In our work, the development of such platform is therefore accomplished through the web technologies like, e.g., HTML, Java/JavaScripts, MySql.

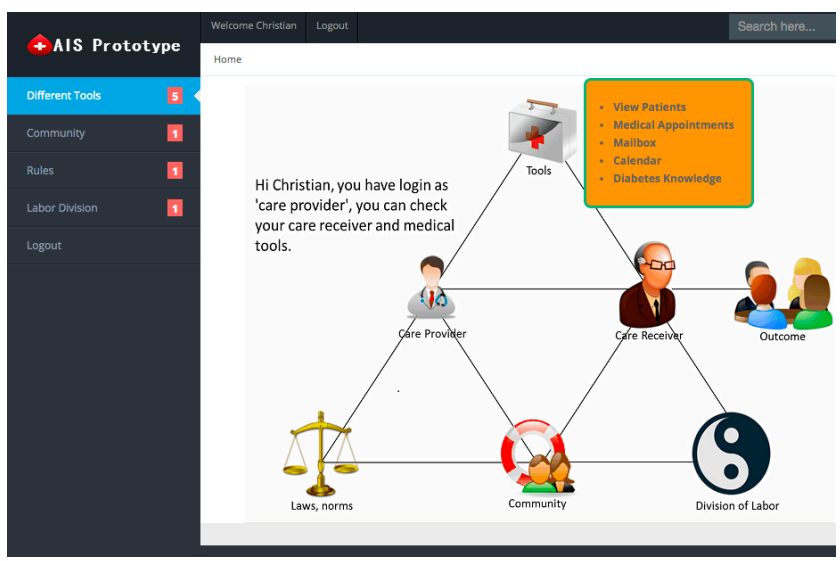

Fig. 3. Example webpage for the care provider using the AIS prototype

For a particular AIS user, once the user is successfully registered in the system, he/she is assigned with a role like, e.g., a care provider, a care receiver. Then, the AIS system shows the particular webpage content in accordance with the user's role. For example, Fig. 3 shows that the user takes the 
role as a doctor. Moreover, different roles may share the same activity theory ontology in terms of the same information and responsibility about the targeted activity units.

\section{B. System Deployment}

By the end of 2015, the developed prototype is practically deployed in the Zhengzhou University Hospital for the experimental test purposes. The reason for the selection of the city Zhengzhou is that, the average age of elderly people in Zhengzhou is above the one in other major cities in China. During the test, the deployed system is available for the use by both the healthcare providers (e.g., the doctors working in the hospital) and the healthcare receivers (e.g., the elderly people staying at home), as shown in Fig. 4. Examples of their interactivities are booking an appointment with a doctor, reporting outcomes by either nurses or by doctors, checking results by either patients or by the patients' relatives.

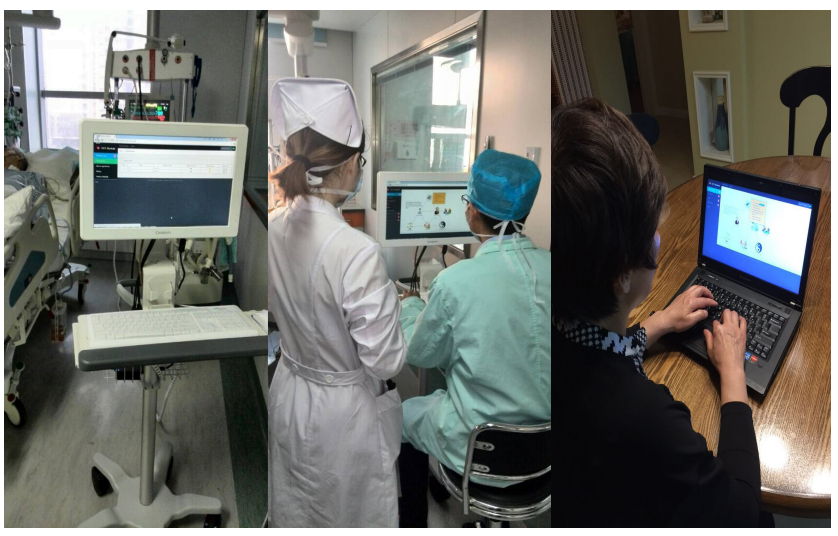

Fig. 4. Deployment of the AIS prototype in Zhengzhou University Hospital

\section{Survey Evaluation and Discussions}

To study the feasibility and usability of the suggested system (i.e., AIS), the survey based system evaluation is conducted. The evaluation procedure consists of two parts. The first part is regarding the questionnaire distribution to the AIS users. Specifically, two different groups of questionnaire are distributed to the healthcare providers and the healthcare receivers, respectively. The second part is to collect and analyze the questionnaire results.

Specifically, for the healthcare providers, there are 60 people participating in the survey with the given questionaries. These participants are the doctors, nurses and the patient relatives. Similarly, the questionaries for health receivers is distributed to 100 patients, and 86 of them are elderly people. Eventually, 55 results are received from the health providers, while 87 results are received from the health receivers. In other words, the response rates to the survey are around $92 \%$ and $87 \%$ for the care providers and receivers, respectively. The discussions about these results are describe below.

On one hand, the number of healthcare providers showing the interest in AIS is around $80 \%$. The majority responds related to the interest are referred to as the convenience of using
AIS to deal with the task scheduling. For instance, i) doctors arrange appointments to meet patients, ii) nurses prepare the patients' outcomes for both doctors and the patients' families, iii) the family members of a patient periodically track the health status of this patient. However, two important problems are also addressed in the survey results. The first problem is that it is difficult for the healthcare providers to assist the emergent healthcare by using current AIS. The second problem is that some doctors prefer a face-to-face meeting with the particular patients who have serious illness, in order to alleviate their psychological pressures.

On the other hand, around $85 \%$ of patients think that AIS is helpful for them to do self-care. However, only $50 \%$ of patients are sure about that they can get benefits from AIS. The reason for this result is that most patients are elderly people, and they are not familiar with web technologies. This motivates us to do further development on AIS by making the simple client running on smartphones.

\section{CONCLuSion}

The problem of coping with inter-activity among different health actors for knowledge sharing in eHealth is considered. The activity theory based ontology model is suggested for representing the health actors existent in eHealth. Three typical inter-activities among these health actors are presented. A prototype software system Activity Information System (AIS) is developed to enhance the inter-activities in the eHealth area. The system is practically deployed for evaluation purposes. The survey based evaluation is conducted, and the results show the feasibility and usability of the developed system. The future work is to do the further performance evaluation on the AIS, and integrate the machine-to-machine techniques such as the connected smart home into the AIS.

\section{REFERENCES}

[1] ACTIVE A.G.E. Urbact II Thematic Network, "Prague Local Action Plan: Age and care", December, 2011.

[2] C.C.Y. Poon, Q. Liu, H. Gao, W.H. Lin, and Y.T. Zhang, "Wearable Intelligent Systems for E-Health ", Journal of Computing Science and Engineering, vol. 5, no.3, pp. 246-256, 2011.

[3] M. Hemam, and Z. Boufaida, "Towards a Multi-Viewpoints Ontological Model for Knowledge Sharing in E-health", International Journal of Information Science and Intelligent System, vol. 3, no.3, pp. 5-14, 2014.

[4] T. Widmer, M. Premm, M. Schuele, J. Murray, and P. Karaenke, "Distributed Knowledge Sharing For Patient Guidance eHealth Services", 21st European Conference on Information Systems, Utrecht, Netherlands, 2013.

[5] G.H. Bai, and Y. Guo, "Activity Theory Ontology for Knowledge Sharing in E-health", Internationl Forum on Information Technology and Application, Kunming, China, 2010.

[6] Engeström, "Learning by Expanding, An Activity-Theoretical Approach to Developmental Research", Helsinki: Orienta-Konsultit, Finland, 1987.

[7] L. S. Vygotsky, "Mind in Society:The Development of Higher Psychological Processes", Harvard University Press, Cambridge, MA, 1978.

[8] A.N. Leontev, "Activity, Consciousness and Personality", PrenticeHall: Englewood Cliffs, NJ, 1978.

[9] Engeström, "Working, and Imagining: Twelve Studies in Activity Theory", Helsinki: Orienta-Consultit Oy, Finland, 1990. 